\title{
Modeling of III-V-based Nanohole Filling
}

\author{
Antal Ürmös, Zoltán Farkas, Ákos Nemcsics
}

Institute of Microelectronics and Technology, Óbuda University, Tavaszmező utca 17, H-1084 Budapest, Hungary; e-mails: urmos.antal@phd.uni-obuda.hu, farkas.zoltan@kvk.uni-obuda.hu, nemcsics.akos@kvk.uni-obuda.hu

Abstract: It is well-known, that several areas of science (for example electronics) have been revolutionized by the application of nano structures. For this reason, it is important to simulate the evolution of these nano structures. The III-V-composite semiconductor based nanohole backfilling is modeled in this paper. The backfilling is described with the viscosity of the liquid gallium, instead of modeling of the real atomic displacements. The construction of the model begins with the macroscopic interpretation of the viscosity. In order to model the atomic displacement at microscopic level, the microscopic viscosity was introduced. It is shown, that under certain conditions we get back the original ArrheniusAndrande equation from the microscopic viscosity model. During the simulation, both the viscosity and the equilibrium height of the backfilled nanohole was determined as a function of temperature.

Keywords: GaAs; nanohole backfilling; viscosity; modeling

\section{Introduction}

It is well-known, that several areas of science were revolutionized by the application of nano structures. Recently, increasing proportion of electronic devices containing nanostructures are found.

One excellent example is the III-N-based semiconductor devices, for example the GaN-based blue LEDs. The active regions of these devices consist one or more InGaN quantum wells. These wells are inserted between thicker GaN layers, in a sandwich-like manner. By topologically changing the $\mathrm{InN}$ and $\mathrm{GaN}$ regions in the InGaN wells, the wavelength of emitted light extends from the ultraviolet to the color of amber. Another possibility is the application of quantum dots, instead of quantum wells. In this case, the large light intensity of the LED is generated by InGaN quantum dots with the aid of phase separation effect [1].

Another example is the nanostructured solar cells. The efficiency of these cells is significantly influenced by the electronic band structure of the semiconductor. A good solution is the application of various nanostructures such as quantum dots and quantum wells [2-4] for increasing of the efficiency of the structure. 


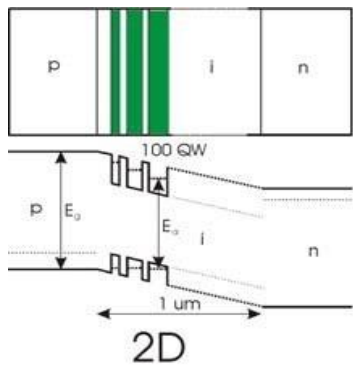

a.

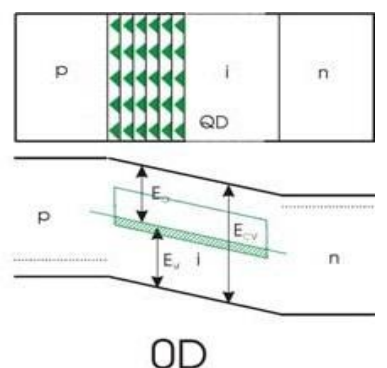

b.

Figure 1

The efficiency of the solar cells can be increased with the application of the multiple quantum wells (a) and quantum dots (b) (source: [3])

With the application of these structures, the solar cells can utilize wider wavelength range of the solar spectrum. Efficiencies of over $40 \%$ can be achieved with multilayer solar cells that contain quantum dots. These devices operate on the principle of tunnel effect. In the case of another nano structured solar cells containing quantum wells, up to $40 \%$ efficiency can be achieved through the utilization of tunnel effect (Figure 1a). This excellent solar cell efficiency can be surpassed by the usage of quantum dots (Figure 1b). In this case, intermediate energy levels are created in the energy band gap of the semiconductor. This structure is able to utilize the energy of photons, the energy of which differs from the energy determined by gap of the original semiconductor. This way, an efficiency of $60 \%$ can be achieved.

An important requisite for the fabrication of the GaAs-based nanostructures, presented in this chapter, is the controlled growth of semiconductor crystal layers and nanostructures. Primarily, these thin, several monolayer thick layers and other nano objects can be grown with the method of molecular-beam-epitaxy (MBE) [5] [6].

\section{The Droplet Epitaxy}

The various nanostructures can be fabricated with several growth method. There are three growth regimes such as the traditional Frank Van Der Merve, VolmerWeber and Stransky-Krastanov methods. The difference among these methods, is the difference of the bonding energies between atom-substrate and the atomneighboring atom relations. In the case of Frank Van Der Merve growth, the atomsubstrate bonding energy is bigger than the atom-neighboring atom bonding energy, so mainly two dimensional layers are formed. In case of Volmer-Weber growth the atom-substrate bonding energy is smaller than the atom-neighboring atom bonding energy, so clusters are primarily formed. In case of StranskyKrastanov growth the adatom-substrate bonding energy and the adatom- 
neighboring atom bonding energy are comparable, so both clusters and layers can be formed.

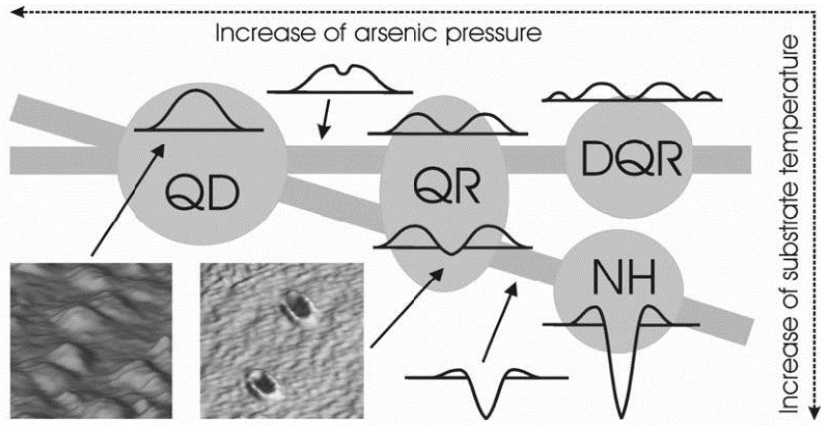

Figure 2

The development of different nanostructures in the function of the temperature of the sample and the pressure of the remaining gas (source: [11])

With the aforementioned methods, only layers or clusters or dots can be formed. A novel technique, called droplet epitaxy allow us to create not only quantum dots but also quantum rings, double quantum rings and nanoholes. The conception of the droplet epitaxy was developed by Koguchi and his co-workers, in the beginning of the 1990 years [7, 8, 9, 10]. The droplet epitaxy is a two stages technology. Firstly a metal droplet of the III. main group of the periodic system (for example $\mathrm{Ga}$ ) is deposited onto the substrate surface (for example GaAs). After that metallic nano-clusters are created, the final shape which is determined by the sample temperature and by the gas pressure of the residual main group $\mathrm{V}$ element (for example arsenic). In this way, various nanostructures can be developed (Figure 2).

As it can be seen on Figure 3, the nanoholes are formed in high temperature and minimal arsenic pressure [12]. The formation of the nanoholes are the following: a gallium droplet is deposited to the substrate surface. Because of the concentration gradient, the arsenic atoms diffuse into the gallium droplet. In the same time, the gallium atoms leave the droplet in the manner of surface diffusion. Finally, the nanohole forms. The process is explained in the Figure 3.

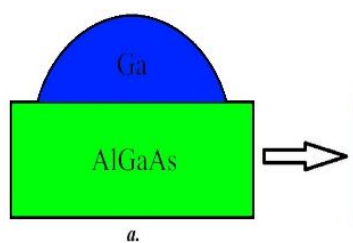

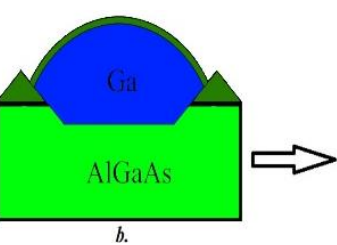

Figure 3

The formation of the nanoholes (source: [12]). The formation of the nanoholes are the following: a gallium droplet are deposited to the substrate $(\boldsymbol{a})$. The arsenic atoms move to the droplet and the same time the gallium atoms leave the droplet $(\boldsymbol{b})$. Finally nanohole forms $(\boldsymbol{c})$ 
The variables of Figure 3/c are explained below: $r_{0}$ is the outer radius (nanohole and the ring), $h_{w}$ is the height of the ring, $d_{H}$ is the depth of the nanohole. If the nanoholes, formed on this way, were filled with metal atoms (for example gallium), then we talk about inverted quantum dots. There are 4 types of inverted quantum dots: type 1 quantum dots are the white light emitters, type 2 quantum dots are the uniform quantum dots, type 3 quantum dots are the ultra low-density quantum dots, type 4 quantum dots are the vertically stacked quantum dot molecules (Figure 4) [12].

In Figure 4, Type 1 quantum dots (white light emitters) are shown which were fabricated by local droplet etching on AlGaAs surface at $\mathrm{T}=550-620{ }^{\circ} \mathrm{C}$ temperature. Bimodal, shallow and deep nanoholes are formed with this method. During the backfilling process of the nanoholes, the shallow ones are fully and the deep ones are partially filled. Local droplet etching creates Type 2 (uniform) forms (Figure 4b) and the density of shallow holes reduced at elevated temperature. These quantum dots are partially buried and their size accurately controlled through the amount of the deposited GaAs. The ultra-low density (ULD, Type 3) quantum dots, filled with GaAs, can be characterized with even lower density and deeper holes (Figure 4c). Figure 4d displays vertically stacked quantum dot molecule, which consists of two closely placed quantum dots. This is the simplest interacting system, made of nanostructures. This structure contains two double filled ultra-low density quantum dots. The two quantum dots are separated with two well-defined AlGaAs barriers.

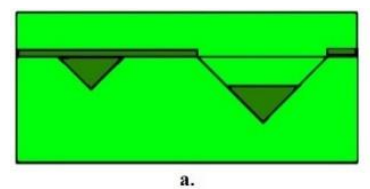

a.

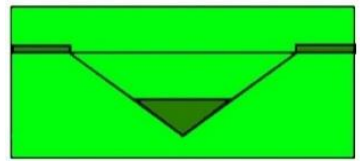

c.

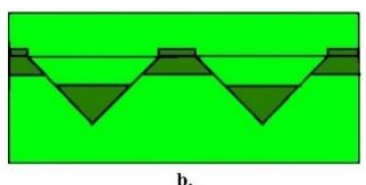

b.

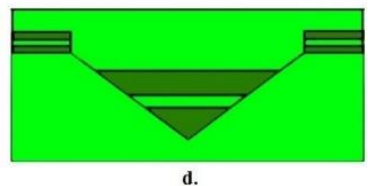

Figure 4

Formation of the inverted quantum dots. The a) is the group of Type 1 quantum dots, the $\mathbf{b}$ ) is the group of Type 2 quantum dot, the c) is the group of Type 3 quantum dots and $\mathbf{d}$ ) is the vertically stacked quantum dot molecule (source: [12]).

\section{The Simulation Algorithm}

In the simulation, the initial state is the nanohole, showed in the Figure 5 [13]. "A" denotes the area adjacent to the ring, " $\mathbf{B}$ " denotes the ring and " $\mathbf{C}$ " is the nanohole. The $D_{\text {ring }}$ is the width of the ring (radius of outer circle of the ring less 
radius of inner circle of the ring), the $D_{\text {hole }}$ is the diameter of the hole, the $L$ is the height of the ring above the substrate surface, the $H$ is the height of the ring above the bottom of the nanohole. The angle $\alpha$ is the half angle of the orifice of the nano hole, the value of which value is $55^{\circ}$.

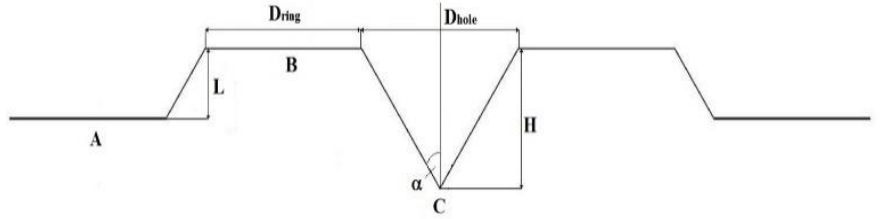

Figure 5

The base structure of the nanohole. The point " $\mathbf{A}$ " is the area next to the ring, the point "B" is the ring and the point "C" is the nanohole. A $D_{\text {ring }}$ is the width of the ring, a $D_{\text {hole }}$ is the diameter of the hole, the $L$ is the height of the ring above the surface of the substrate, the $H$ is the height of the ring above the bottom of the nanohole. The $\alpha$ angle is the half angle of the orifice of the nano hole.

During the simulation, in the first step, Ga was deposited to the substrate surface (Figure 6). In this chapter, the ideal case is investigated, when there is no surface diffusion, meaning that the deposited atoms do not migrate on the surface.

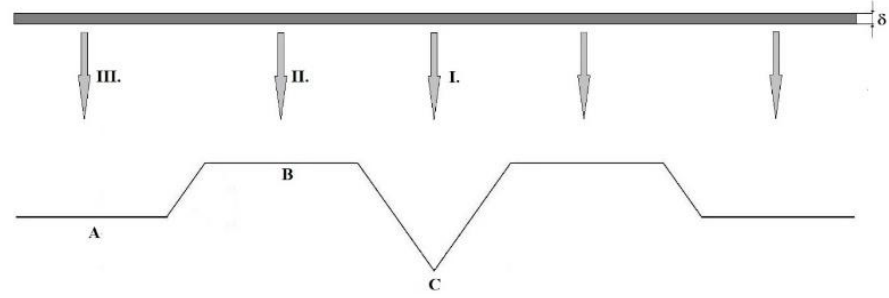

Figure 6

The parts of the volume, that get to different parts of the nanohole, during the deposition (base layer)

From the volume of deposited layer, the volume I goes directly to the nanohole $\left(V_{I}\right)$. The volume that is deposited directly to the nanohole can be obtained from the followng equation:

$V_{I .}=r_{\text {hole }}^{2} * \pi * \delta$

where the $r_{\text {hole }}$ is the radius of the nanohole and $\delta$ is the thickness of the deposited layer. The height of the newly deposited volume in the cone can be computed by the cone volume formula:

$m=\sqrt[3]{\frac{3 * V_{I}}{\pi * g^{2} \alpha}}$

where the $m$ is the backfilled height from the point of the cone and the $V_{I}$ is the volume gets directly into the nanohole. The volume $I I$, is the additional ring 
volume, the volume $I I I$ is the volume that is deposited aside the ring, $\delta$ denotes the thickness of the layer (Figure 7).

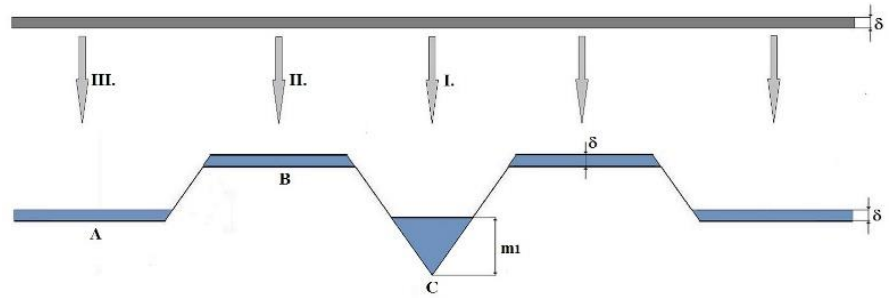

Figure 7

The parts of the volume, that get to different parts of the nanohole, during the deposition (first layer).

The heights on the ring and next to the ring is $\delta$.

In the course of the second step another layer is deposited, the thickness of which is also $\delta$ (Figure 8). The volumes in the ring and aside ring to the ring are equal, and the monolayer thickness will be also $\delta$. There are two ways to compute the filled height in the nano hole. According to the first method, we compute it with the volume of the cone, which is the following:

$$
m_{2}=\sqrt[3]{\frac{3 *\left(V_{I .1}+V_{I .2}\right)}{\pi * \operatorname{tg}^{2} \alpha}}
$$

where $m_{2}$ is the height backfilled in the second step, a $V_{I .1}$ is the backfilled volume in the first step and $V_{I .2}$ is the backfilled volume in the second step. This formula can be generalized, in the following way:

$m_{i}=\sqrt[3]{\frac{3 * \sum_{k=1}^{i} V_{I . k}}{\pi * g^{2} \alpha}}$

where $m_{i}$ is the backfilled height in the $i$-th step and $\sum_{k=0}^{i} V_{I . k}$ is the the sum of the volume deposited in $i$-th step and the previous steps.

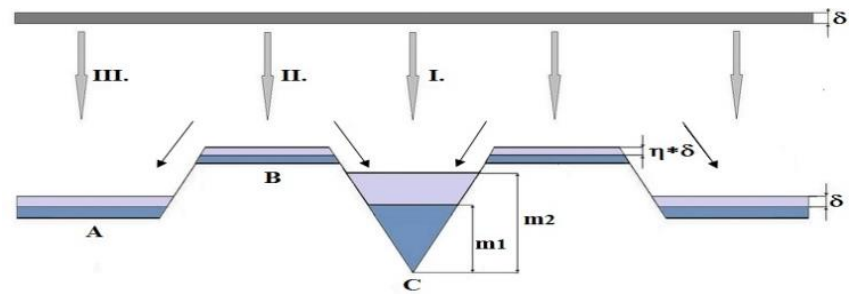

Figure 8

The parts of the volume, that get to different parts of the nanohole, during the deposition (second layer). The heights on the ring and next to the ring is $\delta$.

Another possible solution is, that we can compute the height of the truncated cone, solving a third order equation. This equation is as follows: 
$m_{k}^{3} *\left(\frac{\pi}{3} * \operatorname{tg}^{2} 55\right)+m_{k}^{2} *\left(\pi * r_{k-1} * \operatorname{tg} 55\right)+m_{k} *\left(\pi * r_{k-1}^{2}\right)-V_{k}=0$

where $m_{k}$ is the height of the truncated cone, $r_{k-1}$ is the radius of the circle of the top of the deposited material within the cone, computed in the previous step, the $V_{k}$ is the volume of the truncated cone, which is equal to the volume $V_{I . k}$, which gets directly to the nanohole in the $k$-th step. In the next step, this height is added to the total sum of the backfilled heights, computed in the previous steps:

$m_{i}=\sum_{k=1}^{i} m_{k}$.

The ring volume $V_{I I}$. can be computed, that the volume of the inner truncated cone $\left(V_{i t c}\right)$ is subtracted from the total volume (which is the sum of the volume of the inner truncated cone $\left(V_{i t c}\right)$ and the ring volume $\left.V_{I I .}\right)$. The $V_{i t c}$ volume of the inner truncated cone can be calculated by the following formula:

$V_{i t c}=\frac{\pi}{3} m_{i t c}\left(R_{i t c}^{2}+r_{i t c}^{2}+R_{i t c} * r_{i t c}\right)$

where $R_{i t c}$ is the larger radius, the $r_{i t c}$ is the smaller radius and $m_{i t c}$ is the height of the inner truncated cone. The $r_{i t c}$ is the smaller radius can be computed by the following formulae:

$r_{i t c}=H * \operatorname{tg} \alpha$

where $H$ is the depth of nanohole, the $\alpha$ is the half angle of the orifice of the nanohole (its value is $55^{\circ}$ ). The $R_{i t c}$ is the larger radius can be computed by

$R_{i t c}=H * \operatorname{tg} \alpha+m_{i t c} * \operatorname{tg} \alpha$

formula, where $H$ is the depth of nanohole, the $m_{i t c}$ is the height of the inner truncated cone and the $\alpha$ is the half angle of the orifice of the nanohole (its value is $\left.55^{\circ}\right)$. As a consequence, the volume of the inner truncated cone:

$V_{i t c}=m_{i t c}^{3} *\left(\frac{\pi}{3} * \operatorname{tg}^{2} \alpha\right)+m_{i t c}^{2} *\left(H * \pi * \operatorname{tg}^{2} \alpha\right)+m_{i t c} *\left(H^{2} * \pi * t g^{2} \alpha\right)$.

Figure 9 illustrates the parameters. $R_{i t c}, r_{i t c}, m_{i t c}$ are the larger radius, the smaller radius and the height of the inner truncated cone respectively. The $\alpha$ is the half angle of the orifice of the nanohole (its value is $55^{\circ}$ ), $H$ is the depth of nanohole and the $y$ an auxiliary variable, the value of which is $y=m_{i t c} * \operatorname{tg} \alpha$.

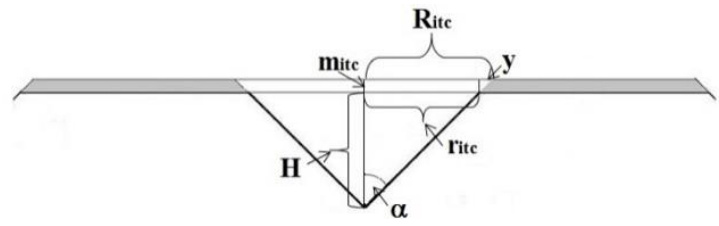

Figure 9

The interpretation of the parameters. In the figure $R_{i t c}$ is the larger radius, the $r_{i t c}$ is the smaller radius and $m_{i t c}$ is the height of the inner truncated cone, the $\alpha$ is the half angle of the orifice of the nanohole (its value is $55^{\circ}$ ), $H$ is the depth of the nanohole and the $y$ an auxiliary variable, the value of which is

$$
y=m_{i t c} * \operatorname{tg} \alpha \text {. }
$$


The total volume $\left(V_{t c}\right)$ can be computed by the following formula:

$V_{t c}=\frac{\pi}{3} m_{t c}\left(R_{t c}^{2}+r_{t c}^{2}+R_{t c} * r_{t c}\right)$

where $R_{t c}$ is the greater radius, $r_{t c}$ smaller radius, az $m_{t c}$ the height of the total truncated cone. The $r_{t c}$ smaller radius can be computed by the following formula

$r_{t c}=H * \operatorname{tg} \alpha+d-m_{t c} * \operatorname{tg} \alpha$

where $H$ is the depth of the nanohole, $m_{t c}$ the height of the truncated cone and $\alpha$ is the half angle of the orifice of nanohole (its value is $55^{\circ}$ ). The $R_{t c}$ bigger radius can be computed by the following formula:

$R_{t c}=H * \operatorname{tg} \alpha+m_{t c} * \operatorname{tg} \alpha$

where $H$ is the depth of the nanohole, $m_{t c}$ the height of the complete truncated cone (in this case it is the thickness of the deposited layer) and $\alpha$ is the half angle of the orifice of the nanohole (the value of which is $55^{\circ}$ ). As a consequence, the volume of the truncated cone:

$$
\begin{aligned}
& V_{t c}=m_{t c}^{3} *\left(\frac{\pi}{3} * \operatorname{tg}^{2} \alpha\right)-m_{t c}^{2} *\left(d * \pi * \operatorname{tg} \alpha-H * \pi * \operatorname{tg}^{2} \alpha\right)+m_{t c} * \\
& \left(d^{2} * \pi+2 * d * H * \pi * \operatorname{tg} \alpha+H^{2} * \pi * \operatorname{tg}^{2} \alpha\right)
\end{aligned}
$$

where $H$ is the depth of the nanohole, $m_{t c}$ the height of the truncated cone (in this case is the thickness of the deposited layer) and $\alpha$ is the half angle of the orifice of the nanohole (its value is $55^{\circ}$ ).

Figure 10 illustrates the parameters. $R_{t c}, r_{t c}, m_{t c}$ are the greater radius, the smaller radius and the height of the inner truncated cone, respectively. The $\alpha$ is the half angle of the orifice of the nanohole (its value is $55^{\circ}$ ), $H$ is the depth of the nanohole, the $d$ is the lower plateau of the ring and the $x$ is the upper plateau of the ring.

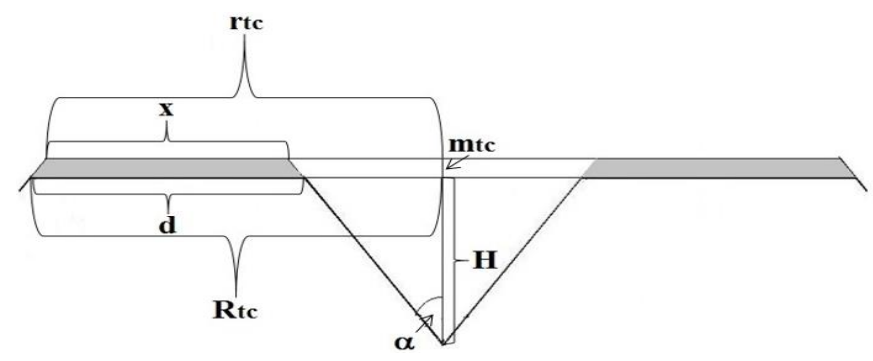

Figure 10

The interpretation of the parameters. In the figure $R_{t c}$ is the larger radius, the $r_{t c}$ is the smaller radius and $m_{t c}$ is the height of the inner truncated cone, the $\alpha$ is the half angle of the nanohole (its value is $\left.55^{\circ}\right), H$ is the depth of the nanohole, the $d$ is the lower plateau of the ring and the $x$ is the upper plateau of the ring. 
Taking into consideration the parameters defined above, the $V_{I I}$. ring volume can be easily described by the following formula:

$V_{I I .}=V_{t c}-V_{i t c}$

where $V_{t c}$ is the volume of the complete truncated cone, a $V_{i t c}$ is the volume of the inner truncated cone. This substraction takes the form (with substituting the respective/appropriate parameters):

$V_{I I .}=-m_{\text {ring }}^{2} *\left(d * \pi * \operatorname{tg} \alpha+2 * H * \pi * \operatorname{tg}^{2} \alpha\right)+m_{\text {ring }} *\left(d^{2} * \pi+2 * d *\right.$

$H * \pi * \operatorname{tg} \alpha$ )

where $H$ is the depth of the nanohole, $m_{\text {ring }}$ the height of the ring (in this case is the thickness of the deposited layer) and $\alpha$ is the half angle of the orifice of the nanohole (its value is $55^{\circ}$ ), the $d$ is the width of the lower plateau of the ring.

\section{The Mechanism of the Atomic Displacement}

In the reality, at finite temperature $\left(n * k * T>E_{\text {bonding }}\right)$ the atoms migrate on the surface. The parameters of migration depend on the temperature. This surface migration can be modeled by many methods, for example Kinetic Monte-Carlo method $[14,15]$. In this paper, instead of this algorithm the dynamic viscosity of liquid gallium will be applied. This is an approximation, and this attribute originates from the macroscopic description. The viscosity is the function of the temperature. From macroscopic point of view, the dynamical viscosity is a proportionality factor, depends on the properties of the liquid material. Dynamic viscosity is the ratio of shear stress to shear velocity:

$\tau=\eta * \frac{d \gamma}{d t}$

where $\tau$ is the shear stress, $\eta$ is the dynamic viscosity, $\frac{d \gamma}{d t}$ is the shear velocity. In case of liquid metals, the viscosity can be determined in a multiple ways [16-20]. In this paper, the Arrhenius-Andrande equation is used [20], its formula is the following:

$\mu(T)=\mu_{0} * e^{\frac{E}{R * T}}$.

In this formula, the $\mu(T)$ is the dynamic viscosity, the $\mu_{0}$ is a pre-exponential factor (in case of gallium its value is 0.436 ), $E$ is the activation energy (its value in case of $\mathrm{Ga}$ is $4000 \frac{\mathrm{J}}{\mathrm{mol}}$ ), the $R$ is universal gas constant (its value is $8,3144 \frac{\mathrm{J}}{\mathrm{K} * \mathrm{~mol}}$ ) and the $T$ is the temperature (on Kelvin scale).

In the microscopic approach the probability of the occurrence of the event is given at atomic level in order to determine the dynamic viscosity [15, 21]: 
$P\left(E_{a}, T\right)=e^{-\frac{E_{a}}{R * T}}$

where $E_{a}$ is the activation energy of the given event, $T$ is the temperature (on Kelvin scale), $R$ is the universal gas constant (its value is $8,3144 \frac{\mathrm{J}}{\mathrm{mol} * K}$ ). The $k$ hopping frequency is calculated by this formula:

$k=k_{0} * e^{-\frac{E a}{R * T}}$

where $k_{0}$ is the atomic vibration frequency. Parameter $k_{0}$ can be determined in the subsequent way:

$k_{0}=\frac{2 * k_{B} * T}{h}$

where $k_{B}$ is the Boltzmann constant, $h$ is the Planck constant. After this step the $\rho=\rho\left(E_{a}, T\right)$ geometrical factor is introduced, in the following way [26]:

$\rho\left(E_{a}, T\right)=l * e^{\frac{2 E_{a}}{R * T}}$

where $l$ is a scalar value, but it can be both a single variable or a multivariable function. Let $E_{0}=E_{a}[n]$, where $E_{0}$ is the bulk activation energy, $E_{a}[n]$ an density functional, which gives the binding energy of the atomic multeity, consists of $n$ binding energy, with taking into consideration of atom-atom, atom-electron, electron-electron interactions. Consequently the dynamic viscosity is:

$\mu(T)=\rho\left(E_{0}, T\right) * k=k_{0} * l * e^{\frac{E_{0}}{R * T}}$

where the $k_{0} * l$ product is the $\mu_{0}$ pre-exponential factor. Thus the equation 18 is obtained. The effect of the viscosity is taken into consideration according the subsequent way in this paper: the volume of the ring is multiplied by an $\eta(T)$ volume proportional factor, which can be computed with the next formula:

$\eta(T)=\frac{\mu(T)}{\mu\left(T_{m}\right)}=e^{\frac{E}{R}\left(\frac{T_{m}-T}{T * T m}\right)}$

where $T$ is the temperature (on Kelvin scale), the $\eta(T)=\eta$ is a volume proportional factor, $\mu(T)$ is the temperature dependent dynamical viscosity, $\mu\left(T_{m}\right)$ is the melting temperature dependent dynamical viscosity. According to the formulae above, the $V_{I I}$. ring volume can be computed in the subsequent way:

$V^{\prime}{ }_{I I .}=\eta * V_{I I}$

where $V_{I I}$. is the volume of the ring, $V^{\prime}{ }_{I I}$. volume that remains on the ring and $\eta$ is the viscosity. The thickness of the layer remaining on the ring is proportional with the viscosity, thus $m_{\text {ring }}=\eta * \delta$. As a consequence, if this formula was substituted to the 11 equation, then:

$V^{\prime}{ }_{I I .}=-(\eta * \delta)^{2} *\left(d * \pi * \operatorname{tg} \alpha+2 * H * \pi * \operatorname{tg}^{2} \alpha\right)+(\eta * \delta) *\left(d^{2} * \pi+2 *\right.$

$d * H * \pi * \operatorname{tg} \alpha)$ 
where $\eta$ is the volume proportionality factor, the $\delta$ is the thickness of the deposited layer, $H$ is the depth of the nanohole, the $\alpha$ is the half angle of the orifice of the nanohole (its value is $55^{\circ}$ ), the $d$ is the width of the lower plateau of the ring. As a consequence the volume that moves into the nano hole is a sum of the volume moving directly to the nanohole and that moving indirectly into the nanohole, i.e.:

$V^{\prime}{ }_{I .}=V_{I .}+V^{\prime \prime}{ }_{I}$

where $V_{I}$ is the volume, moving directly to the nanohole and $V^{\prime \prime}{ }_{I I}$ is the volume moving indirectly to the nanohole. The latter originates from the ring. This volume can be computed by the following formula:

$V^{\prime \prime}=\frac{(1-\eta)}{2} * V_{I I}$.

where volume of $V^{\prime \prime}{ }_{I I}$ that gets indirectly from the ring to the hole, and $V_{I I \text {. }}$ is the volume remaining to the ring. The reason of $1 / 2$ factor is, that the half of the volume that leaves the ring flows to the nanohole, and the other half of this volume flows to the area adjacent to the ring.

Similarly as in the previous chapter, a $\delta$ thick layer is deposited also (Figure 11). The arrows show, that $\frac{(1-\eta)}{2}$ percent of the ring volume flows down into the hole and aside the ring. The height of the layer on the ring will be $\eta * \delta$. The height of the volume in the nanohole can be calculated by the volume formula of the cone:

$m=\sqrt[3]{\frac{3 * V^{\prime} I}{\pi * g^{2} \alpha}}$

where $m$ is the backfilled height, $V_{I}^{\prime}$. is the volume that arrives to the nanohole, which can be computed by the formula 4 and 5 .

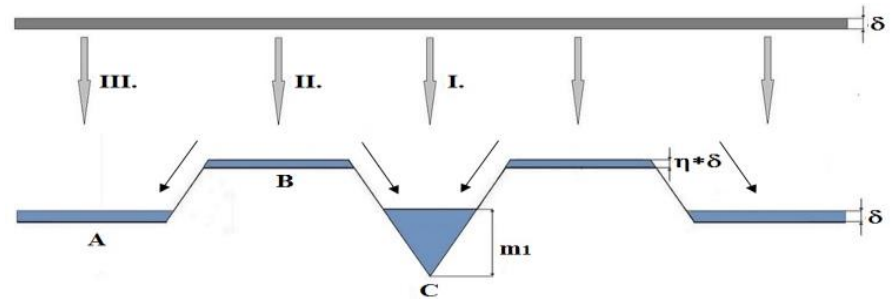

Figure 11

The parts of the volume, move to different parts of the nanohole, during the deposition (first layer). The arrows shows, that $\frac{(1-\eta)}{2}$ percent of the ring volume flows down to the hole and aside the ring. The height of the layer on the ring will be $\eta * \delta$ and aside the ring is $\delta$.

In the second step also a $\delta$ thick layer is deposited (Figure 12). The arrows (similarly as is the previous figure) shows, that $\frac{(1-\eta)}{2}$ percent of the ring volume flows down into the hole and aside the ring. The height of the layer on the ring 
will be $\eta * \delta$ and aside the ring will be $\delta$. The backfilled height in the nanohole, similarly to the previous chapter - can be computed in two different ways. According to the first method, using the volume formula of the cone, the formula of the backfilled height is

$m_{2}=\sqrt[3]{\frac{3 *\left(V^{\prime} I_{I .1}+V^{\prime} I_{I .2}\right)}{\pi * t g^{2} \alpha}}$

where $m_{2}$ is the backfilled height in the second step, the $V_{I .1}^{\prime}$ is the backfilled volume in the first step and the $V_{I .2}^{\prime}$ is the backfilled volume in the second step. This formula can be generalized according to the subsequent way:

$m_{i}=\sqrt[3]{\frac{3 * \sum_{k=1}^{i} V_{I . k}^{\prime}}{\pi * t g^{2} \alpha}}$

where $m_{i}$ is the filled height in the $i$-th step and $\sum_{k=0}^{i} V_{I . k}^{\prime}$ is the the sum of the volumes deposited in the $i$-th and in all of the previous steps. Another possible solution is that with equation 5 a third-order equation is solved and the height of the truncated cone is obtained this way. Afterwards the height is added to the sum of the heights which is in already in the nanohole (equation 6).

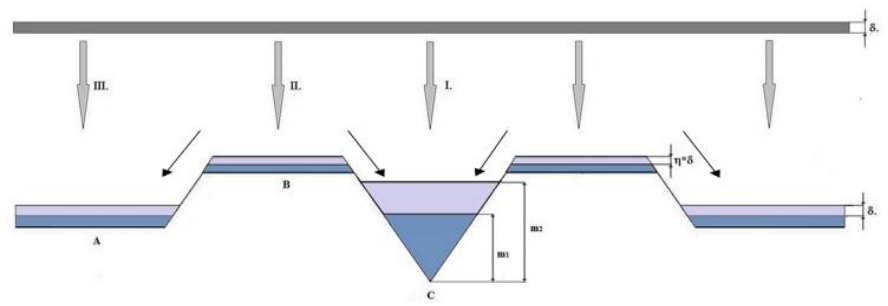

Figure 12

The parts of the volume, goes to different parts of the nanohole, during the deposition (first layer). The arrows shows, that $\frac{(1-\eta)}{2}$ percent of the ring volume flows down to the hole and aside the hole. The height of the layer on the ring will be $\eta * \delta$ and next to the ring is $\delta$.

\section{Discussion}

During the simulation it was investigated as to how the nanohole was filled with GaAs after crystallization. (It means, that the atomic movement described with the help of viscosity of metallic $\mathrm{Ga}$, but the thicknesses calculated from the crystallized GaAs.) The backfilling process (Ga movement) was simulated as the function of the temperature. The height of the ring next to the nanohole increases as the layers are built upon each other. In other words, it was investigated that when the heights of the material inside of the hole and that on the ring became approximately equal. The time was determined as the number of layers already deposited. These two heights considered to be equal, when the differences of the 
top levels of the appropriate structures are closer to each other than unit. The average value of these two heights is called equilibrium height. During the simulation it was our goal to determine, the temperature, where for a given number of layers the height difference between the hole and the ring was minimal. Figure 13 shows the backfilling process of the nanohole. The number of layers already deposited were 1 (denoted with a), 3 (denoted with b), 5 (c) and 7 (d), respectively. If the temperature dependence of the viscosity is neglected, then equilibrium height can be observed at the 22-nd layer. In our example each of the layers contain 2 monolayer thick GaAs deposition. It is important, that in case of GaAs the lattice constant is $0.526 \mathrm{~nm}$, which corresponds 2 monolayers (because this material consists of two components). Here, one monolayer corresponds to 10 pixels, (which is $\frac{0,284}{10}=0.0284 \mathrm{~nm}$ ) in the software, used for the simulation.

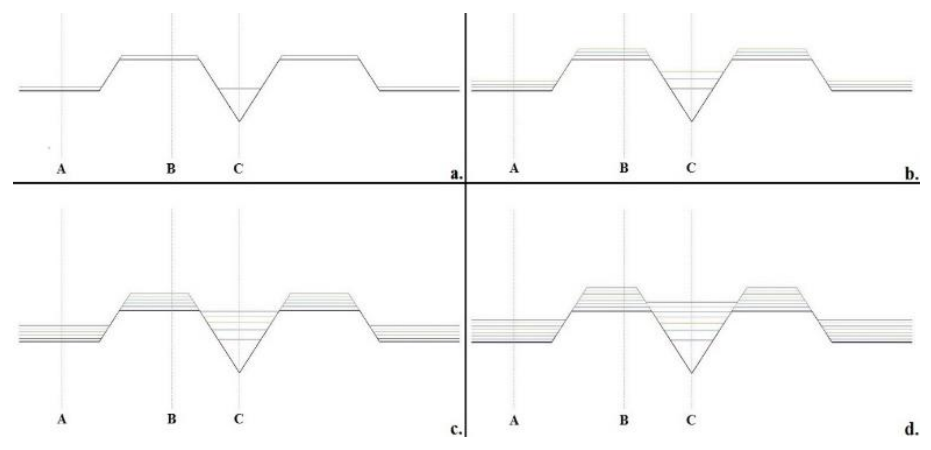

Figure 13

The filling process of the nanohole, in case of 1 (a), 3 (b), 5 (c) and 7 (d) layers

The flow-chart of the simulation algorithm is shown in the Figure 14.

This ideal case exists below $49{ }^{\circ} \mathrm{C}$ substrate temperature. Over this temperature, the equilibrium height and number of layers corresponding to equilibrium height (hereinafter equilibrium number of layers) decrease. As Fig. 15 shows, at $51.6{ }^{\circ} \mathrm{C}$ substrate temperature the nanohole totally filled with the 20 -th layer. At this point the equilibrium height is $15.2945 \mathrm{~nm}$. The bottom line of the charts below represents the filled height in the nanohole (measurement point " $C$ "), while the top line (measurement point " $\mathrm{B}$ ") represent the ring height. The point, where these two lines cross each other is the place of the equilibrium height. The absolute error value is maximal at the layer 18 , its value is 0.0078 (the relative error is $1.561 * 10^{-}$ ${ }^{3} \%$ ). On each parts of chart, the equilibrium height and equilibrium layer pairs are marked with a red lines. It can also be seen that a unit change of the equilibrium height corresponds to increasing change of temperature as the temperature itself rises. Point " $A$ " is selected as reference point (denoting 0 height). 


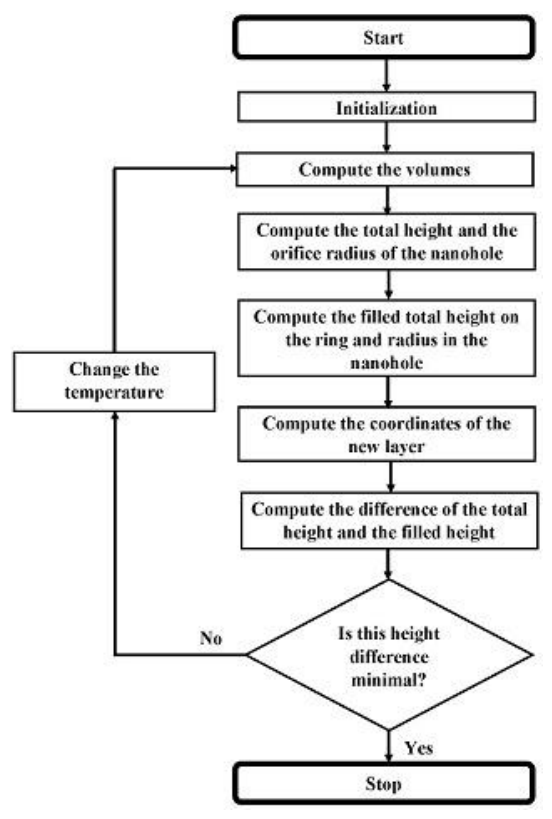

Figure 14

The flowchart of the simulation algorithm
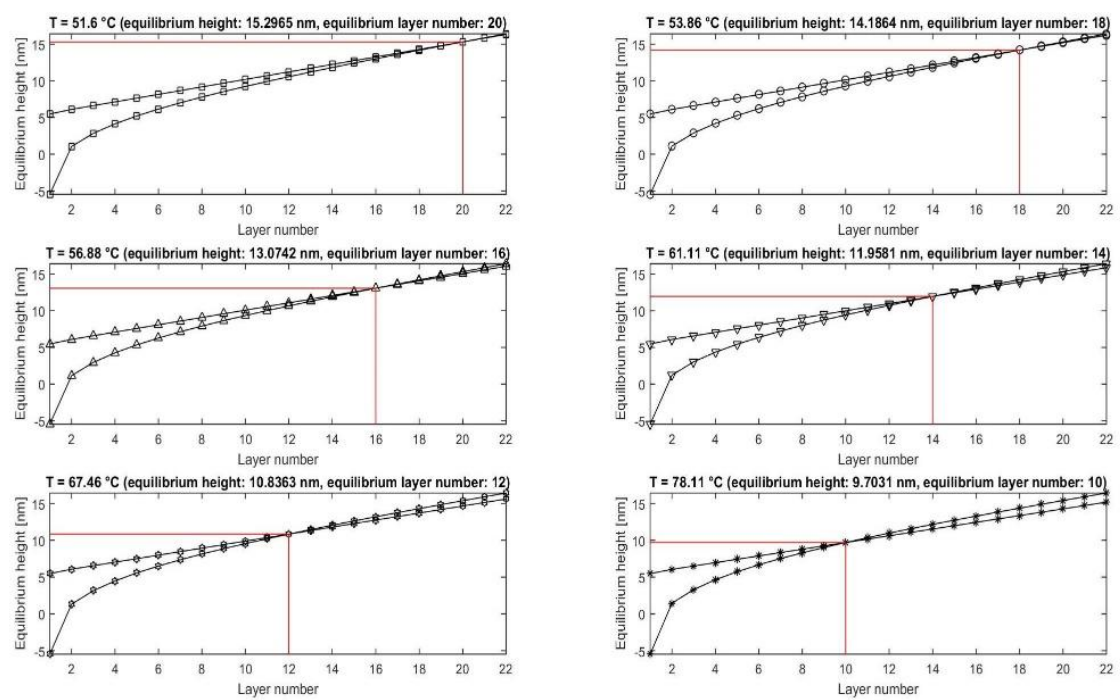

Figure 15

Layer number and equilibrium height diagrams in different temperatures 
On Figure 16 the top diagram shows the equilibrium height as the function of the temperature and the bottom ones shows the equilibrium height as the function of the viscosity.

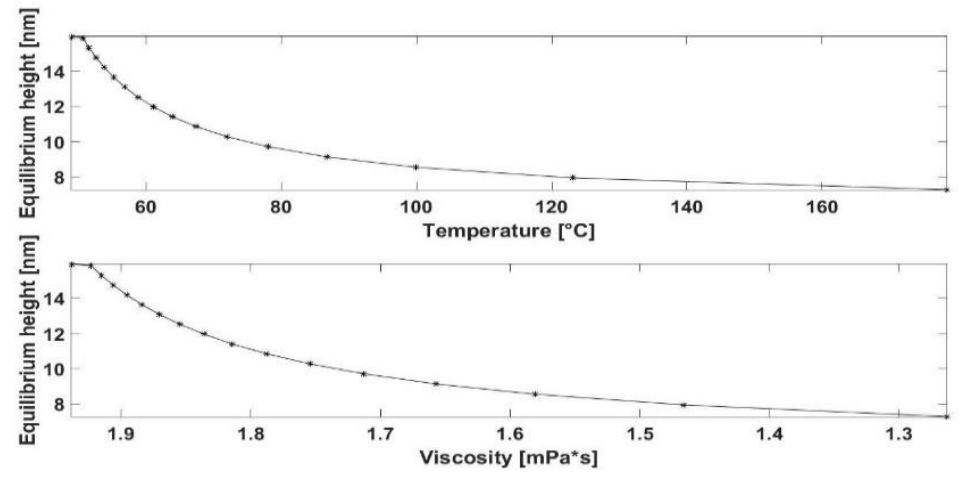

Figure 16

Equilibrium height-temperature (top) and equilibrium height-viscosity (bottom) diagrams

Here, the macroscopic viscosity of $\mathrm{Ga}$ is calculated by Arrhenius-Andrande formula (18 equation). Figure 17 shows the macroscopic temperature-viscosity diagram of Ga.

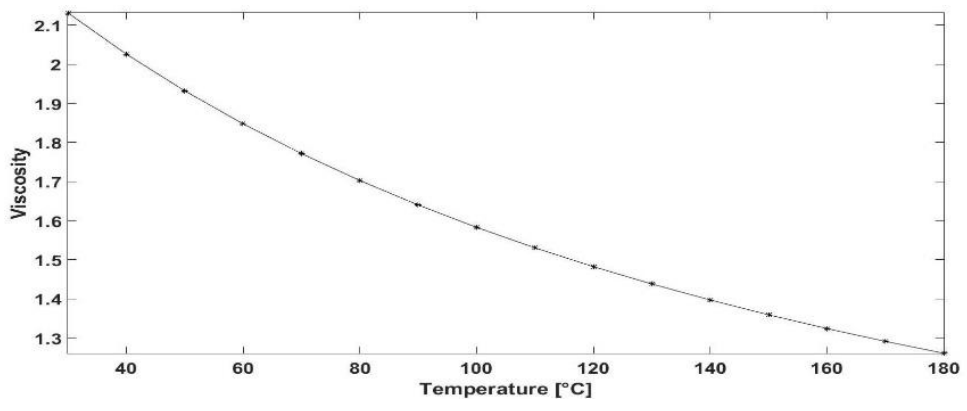

Figure 17

The macroscopic temperature-viscosity diagram of gallium

For the purpose to determine the microscopic temperature-viscosity diagram, first the maximum value of the microscopic viscosity is calculated. For the determination of this, the starting point was the equation 18. At first, the activation energy of the particles is determined, using the following formula [22]:

$E_{p}=E_{0} *\left(1-6 \alpha * \frac{r}{D}\right)$

where the $E_{0}$ the bulk activation energy (its value is in case of gallium $4000 \frac{\mathrm{J}}{\mathrm{mol}}$ ), $E_{p}$ is the cohesive energy of the metal droplets, the $\alpha$ shape factor (in case of sphere shaped particles its value is 1), the $r$ is the radius of the atom, a $D$ is the 
size of particle. Let the melting point of the gallium droplets be $254 \mathrm{~K}\left(-19.15^{\circ} \mathrm{C}\right)$ [23]. The size of the particle is determined by the following formula [24]:

$D=\frac{9 * r * T_{m b}}{T_{m b}-T_{m p}}$

From this equation, in case of $\mathrm{Ga}$, the atomic radius is $1.36 * 10^{-10} \mathrm{~m}$ and the size of the particle (at $254 \mathrm{~K}$ temperature) is $7.5688^{*} 10^{-9} \mathrm{~m}$. If the activation energy is $E_{p}=3568 \frac{\mathrm{J}}{\mathrm{mol}}$ and the particle melting point is $T_{m p}=254 \mathrm{~K}$, then the maximum value of the microscopic viscosity is 2.3626 .

In microscopic level, the temperature-viscosity value pairs of Ga showed in Figure 18 and 19. If one-term power-law is fitted on this value pair series, then the maximum relative error of the fitting is $1.798 \%$, while in case of one-term exponential function the maximum relative error of the fitting is $3.499 \%$. Despite this two-term power and exponential functions are fitting, because the maximum values of the relative error are under $1 \%$. If two-term power law is fitted to the point series (Figure 18), then the temperature-viscosity formula will be the following [25]:

$\mu_{p, M i}(T)=a * x^{b}+c=a * T^{b}+c$

where $\mu_{p, M i}(T)$ is the viscosity as a function of teperature, the $a, b, c$ values are material dependent empirical constants, the $x=T$. Here, the temperature is given in Kelvin. The value of $a$ is $7.766 * 10^{5}, b$ is -2.292 and $c$ is -0.7616 . The upper part of the figure is the power function, fitted to the point series, the bottom graph of the figure shows the error values of the function fitting in the individual points. The maximum value of the fitting error is 0,0008515 (the relative error is $0.0523 \%)$, which is at $123.15^{\circ} \mathrm{C}\left(396.3{ }^{\circ} \mathrm{K}\right)$ temperature.
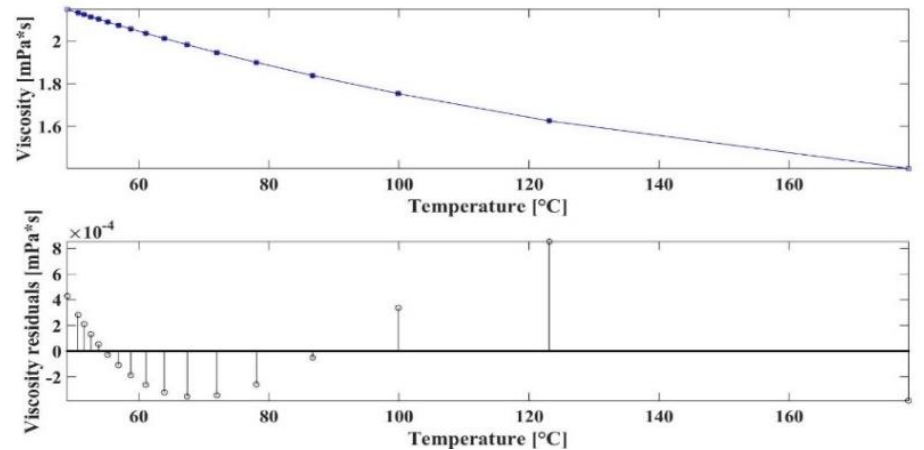

Figure 18

The temperature-viscosity diagram of gallium in microscopic level. Power function is fitted to the point series. The top part of the diagram is the power function, fitted to the point series, the bottom part of the diagram shows the residuals of the function fitting in the individual points. 
If the $a$ coefficient is $k_{0} * l=\tau_{0}$, the $b$ coefficient is 1 , the $c$ coefficient is 0 and the $x=e^{\frac{E_{0}}{R * T}}$, the the equation 18 is obtained. From this we can conclude, that the equation 35 is the microscopic form of equation 18. If exponential sum function was fitted to the point series (Figure 19), then the temperature-viscosity formula would be as follows:

$\mu_{e, M i}(T)=a * e^{b x}+c * e^{d x}=a * e^{b T}+c * e^{d T}$

where $\mu_{e, M i}(T)$ the viscosity as a function of teperature, the $a, b, c$ values are material dependent empirical constants, the $x=T$. The value of $a$ parameter is $41.77, b$ parameter is $-0.01341, c$ parameter is 1.382 and $d$ parameter is 0.001554. The upper part of the diagram is the exponential function fitted to the point series, the bottom graph of the diagram shows the error values of the function fitted to the individual points. The maximum value of the fitting error is 0.0001338 (the relative error is $0.00822 \%$ ), which is at $123.1{ }^{\circ} \mathrm{C}\left(396.3{ }^{\circ} \mathrm{K}\right)$ temperature.
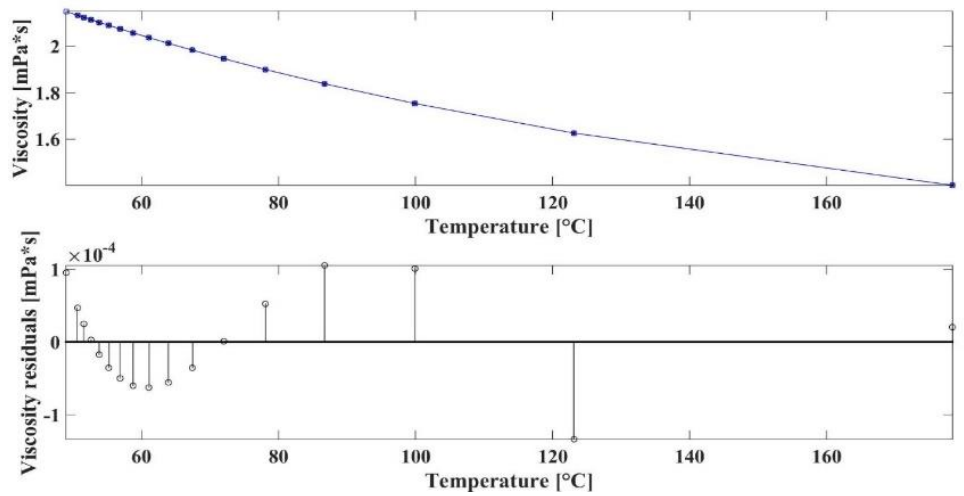

Figure 19

The temperature-viscosity diagram of gallium in microscopic level. Exponential function is fitted to the point series. The top part of the diagram is the power function, fitted to the point series, the bottom part of the diagram shows the residuals of the function fitting in the individual points.

If the $a$ coefficient is $k_{0} * l=\tau_{0}$, the $b$ coefficient is 1 , the $c$ coefficient is 0 and the $x=\frac{E_{0}}{R * T}$, the the equation 18 is obtained. From this we can conclude, that the equation 5.5 is the microscopic form of equation 18 .

The analytical determination of equilibrium height, power function and exponential function are fitted to the temperature-equilibrium height point series. If one-term power function is fitted to this point pair series, then the maximum relative error of the fitting is $36.31 \%$, while in case of one-term exponential function the maximum relative error of the fitting is $39.81 \%$. Two-term power function does not fit at all, if Kelvin scale is used. In spite of this, exponential sum 
function can be fitted well (Figure 20), so the temperature-equilibrium height formula will be the following:

$h_{e}(T)=a * e^{b x}+c * e^{d x}=a * e^{b * T}+c * e^{d * T}$

where $h_{e}(T)$ the equilibrium height in the function of temperature, the $a, b, c, d$ values are material dependent empirical constants, the $x=T$. The value of $a$ parameter is $3.278 * 10^{10}, b$ parameter is $-0.06904, c$ parameter is 16.2 and $d$ parameter is -0.001783 . The top part of the diagram is the two-terms exponential function, fitted to the point series, the bottom part of the diagram shows the value of error of the fitting at individual points. The maximum value of the fitting error is 0.3822 (the relative error is $2.39 \%$ ), which is at $48.95{ }^{\circ} \mathrm{C}\left(322.1{ }^{\circ} \mathrm{K}\right)$.
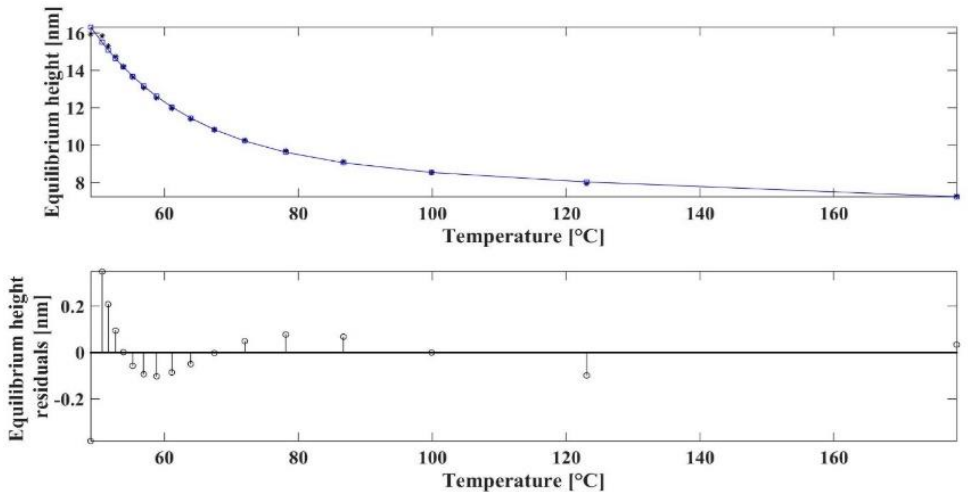

Figure 20

The temperature-equilibrium height diagram of gallium in microscopic level. Exponential function is fitted to the point series. The top part of the diagram is the power function, fitted to the point series, the bottom part of the diagram shows the residuals of the function fitting in the individual points.

\section{Conclusion}

The nanohole backfilling was modeled on a III-V substrate, in this paper. The backfilling was described with the viscosity of liquid gallium, instead of modeling of the real atomic displacements. The crystallization by arsenic was considered as a quick bulk process. During the modeling, we started with the macroscopic interpretation of the viscosity. Microscopic viscosity was introduced in order to enable he presentation of a statistical, microscopic description of the atomic displacement. This was shown for the cases when the original ArrheniusAndrande equation that the microscopic viscosity is returned. During the simulation, the viscosity and the equilibrium height of the filled nanohole was determined as a function of temperature.

\section{References}

[1] G. E. Weng, A. K. Ling, X. Q. Lv, J. Y. Zhang, B. P. Zhang, „III-NitrideBased Quantum Dots and their Optoelectronic Applications," Nano-Micro Lett. 3 (3) pp. 200-207, 2011 
[2] Á. Nemcsics, "Novel Frontiers in Solar Cell Technology", In Hungarian, Elektronikai Technológia, Mikrotechnika, pp. 39-45, 2009

[3] Á. Nemcsics, „Novel Alternative for the GaAs-based Self-organized Quantum-Structure," Óbuda University e-Bulletin, pp. 193-199, 2011

[4] A. Luque, A. Martí, C. Stanley, "Understanding Intermediate-Band Solar Cells" Nature Photonics 6(3):146-152

[5] Á. Nemcsics, I. Réti, V. G. Tényi, P. Kucsera, L. Tóth, P. Harmat, A. Mieville, M. Csutorás, B. Kupás-Deák, T. Sándor, J. Bozsik, "Technological Conditions of the Production Nanostructures by Molecular Beam Epitaxy”, In Hungarian, GÉP LXI, p. 29, 2010

[6] M. A. Herman, H. Sitter, Molecular Beam Epitaxy, Fundamentals and Current Status $2^{\text {nd }}$ ed., Berlin: Springer, 1996

[7] N. Koguchi, S. Takahashi, T. Chikyow, „New MBE Growth Method for InSb Quantum Well Boxes,” Journal of Crystal Growth, pp. 688-692, 1991

[8] N. Koguchi, K. Ishige, „Growth of GaAs Epitaxial Microcrystals on an STerminated GaAs Substrate by Successive Irradiation of $\mathrm{Ga}$ and As Molecular Beams," Japanese Journal of Applied Physics, Vol. 32, Issue 5A, p. 2052, 1993

[9] T. Chikyow, N. Koguchi, „MBE Growth Method for Pyramid-shaped GaAs Micro Crystals on ZnSe(001) Surface Using Ga Droplets," Japanese Journal of Applied Physics, Vol. 29/2, Issue 11, p. 2093, 1990

[10] S. Sanguinetti, N. Koguchi, "Droplet Epitaxy of Nanostructures," in Molecular Beam Epitaxy: From Research to Mass Production, 1st Edition, Waltham, MA, USA, Elsevier Science, 2013, pp. 95-111

[11] Á. Nemcsics, „Growth of GaAs and Related Materials-based NanoStructures by Droplet Epitaxial Method," in Recent Res. Devel. Mat. Sci. Eng., Vol. 4, Kerala, India, Transworld Research Network, 2011, pp. 53-87

[12] C. Heyn, D. Sonnenberg, W. Hansen, „Local Droplet Etching: Selfassembled Nanoholes for Quantum Dots and Nanopillars," in Nanodroplets, New York, Springer Science, 2013, pp. 363-383

[13] Á. Nemcsics, B. Pődör, L. Tóth, J. Balázs, L. Dobos, J. Makai, M. Csutorás, A. Ürmös, „Investigation of MBE Grown Inverted GaAs Quantum Dots," Microelectronics Reliability, Vol. 59, pp. 60-63, 2016

[14] M. Biehl, "Lattice Gas Models and Kinetic Monte Carlo Simulations of Epitaxial Growth," in Birkhäuser Basel, ISNM International Series of Numerical Mathematics, 2004

[15] W. Miller, "Simulation of Epitaxial Growth by Means of Density Functional Theory, Kinetic Monte Carlo, and Phase Field Methods," in Handbook of Crystal Growth: Fundamentals (Volume I, Part A: 
Thermodynamics and Kinetics), $2^{\text {nd }}$ ed., New York, Elsevier B. V, 2015, pp. 521-559

[16] L. Battezzati, A. L. Greer, „The Viscosity of Liquid Metals and Alloys,” Acta Metallurgica, Volume 37, Issue 7, pp. 1791-1802, 1989

[17] C. J. Seeton, „Viscosity-Temperature Correlation for Liquids,” Tribology Letters, Vol. 22, Issue 1, pp. 67-78, 2006

[18] K. E. Spells, „The Determination of the Viscosity of Liquid Gallium over an Extended Nrange of Temperature," Proc. Phys. Soc, 1936

[19] M. J. Assael, I. J. Armyra, J. Brillo, S. V. Stankus, J. Wu,W. A. Wakeham, „Reference Data for the Density and Viscosity of Liquid Cadmium, Cobalt, Gallium, Indium, Mercury, Silicon, Thallium, and Zinc," J. Phys. Chem. Ref. Data, Vol. 41, Issue 3, pp. 033101-1-17, 2012

[20] W. F. Gale, T. C. Totemeier, „General Physical Properties,” in Smithells Metals Reference Book (Eighth Edition), Elsevier Inc., 2004, pp. 1-45

[21] L. Nurminen, A. Kuronen, K. Kaski, "Kinetic Monte Carlo Simulation of Nucleation on Patterned Substrates," Phys. Rev. B, Vol. 63, No. 3, p. 035407, December 2000

[22] W. H. Qi, M. P. Wang, "Size and Shape Dependent Melting Temperature of Metallic Nanoparticles," Materials Chemistry and Physics, Vol. 88, No. 23, pp. 280-284, 2004

[23] G. A. Mansoori, S. A. Rice, "Confined Fluids: Structure, Properties and Phase Behavior," in Advances in Chemical Physics, Vol. 156. Hoboken, NJ, USA: John Wiley \& Sons Inc., 2014, pp. 1-97

[24] D. Xie, M. P. Wang, W. H. Qia, L. F. Cao, "Thermal Stability of Indium Nanocrystals: A Theoretical Study," Materials Chemistry and Physics, Vol. 96, No. 2-3, pp. 418-421, 2006

[25] I. N. Bronshtein, K. A. Semendyayev, G. Musiol, H. Mühlig, „Arithmetic,” in Handbook of Mathematics, Berlin Heidelberg, Springer, 2007, p. 8

[26] L. C. Yang, "Frequency Factor in Arrhenius Decomposition Kinetics for Insensitive Energetic Materials," in American Institute of Aeronautics and Astronautics Inc., 45 ${ }^{\text {th }}$ AIAA/ASME/SAE/ASEE Joint Propulsion Conference \& Exhibit, 2009, pp. 1-28

[27] Z. Erdélyi, M. Pasichnyy, V. Bezpalchuk, J. J. Tomán, B. Gajdics, A. M. Gusak, Stochastic Kinetic Mean Field Model, Computer Physics Communications, Volume 204, July 2016, pp. 31-37, http://dx.doi.org/10.1016/j.cpc.2016.03.003

[28] J. Zheng, W. Zhuang, N. Yan, G. Kou, H. Peng, C. McNally, D. Erichsen, A. Cheloha, S. Herek, C. Shi, Y. Shi, Classification of HIV-I-mediated 
Neuronal Dendritic and Synaptic Damage using Multiple Criteria Linear Programming, Neuroinformatics, Vol. 2, No. 3, pp. 303-326, 2004

[29] R. E. Precup, S. Preitl, M. Balas, V. Balas, Fuzzy Controllers for Tire Slip Control in Anti-Lock Braking Systems, Proceedings of IEEE International Conference on Fuzzy Systems, Budapest, Hungary, Vol. 3, pp. 1317-1322, 2004

[30] S. B. Ghosn, F. Drouby, H. M. Harmanani, A Parallel Genetic Algorithm for the Open-Shop Scheduling Problem using Deterministic and Random Moves, International Journal of Artificial Intelligence, Vol. 14, No. 1, pp. 130-144, 2016

[31] J. K. Tar, J. F. Bitó, I. J. Rudas, Contradiction Resolution in the Adaptive Control of Underactuated Mechanical Systems Evading the Framework of Optimal Controllers, Acta Polytechnica Hungarica, Vol. 13, No. 1, pp. 97121,2016 